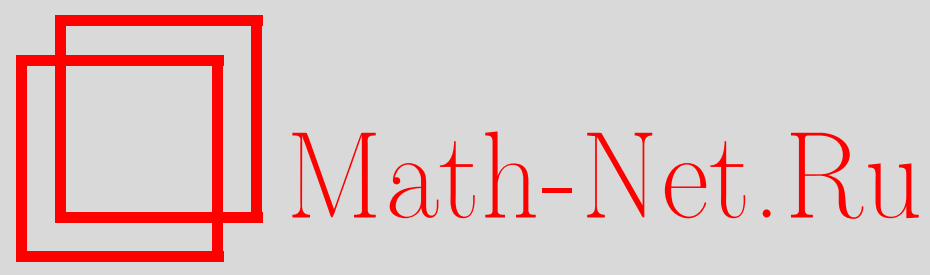

Ю. М. Дюкарев, О критериях неопределенности матричной проблемы моментов Стилтьеса, Матем. заметки, 2004, том 75 , выпуск $1,71-88$

DOI: https://doi.org/10.4213/mzm7

Использование Общероссийского математического портала Math-Net.Ru подразумевает, что вы прочитали и согласны с пользовательским соглашением http://www.mathnet.ru/rus/agreement

Параметры загрузки:

IP: 35.173 .219 .12

26 апреля 2023 г., 11:50:55 


\title{
О КРИТЕРИЯХ НЕОПРЕДЕЛЕННОСТИ МАТРИЧНОЙ ПРОБЛЕМЫ МОМЕНТОВ СТИЛТЬЕСА
}

\author{
Ю.М. Дюкарев
}

\begin{abstract}
В этой статье получены критерии неопределенности матричной проблемы моментов Стилтьеса. Найдены явные формулы для параметров Стилтьеса и исследована мультипликативная структура резольентной матрицы. В неопределенном случае изучены аналитические свойства резольвентной матрицы проблемы моментов. В терминах дробно-линейных преобразований над стилтьесовскими парами дано описание множества всех матриц-функций, ассоциированных с неопределенной проблемой моментов Стилтьеса.

Библиография: 14 названий.
\end{abstract}

1. Введение. Пусть дано целое число $m \geqslant 1$. Символом $\mathbb{C}^{m \times m}$ обозначим множество комплексных квадратных матрищ $m$-го порядка, а символом $\mathbb{C}_{H}^{m \times m}$ - множество эрмитовых матриц $m$-го порядка. Эрмитова матрица $A \in \mathbb{C}_{H}^{m \times m}$ назьвается неотрииательной, если $(f, A f) \geqslant 0 \forall f \in \mathbb{C}^{m}$. Символом $\mathbb{C}_{\geqslant}^{m \times m}$ обозначим множество эрмитовых неотрицательных матриц $m$-го порядка. Неотрицательная матрица $A \in \mathbb{C}_{\geqslant}^{m \times m}$ назьвается строго положительной, если $(f, A f)>0$ для всех ненулевых векторов $f \in \mathbb{C}^{m}$. Символом $\mathbb{C}_{>}^{m \times m}$ обозначим множество эрмитовых строго положительных матриц $m$-го порядка. Кроме того, введем обозначения $\mathbb{R}_{+}=\{t \in \mathbb{R}: t \geqslant 0\}, \mathbb{R}_{-}=$ $\{t \in \mathbb{R}: t<0\}, \mathbb{C}_{+}=\{z \in \mathbb{C}: \operatorname{Im} z>0\}, \mathbb{C}_{-}=\{z \in \mathbb{C}: \operatorname{Im} z<0\}, \mathbb{C}_{ \pm}=\mathbb{C}_{+} \cup \mathbb{C}_{-}$.

В статье рассмотрена матричная проблема моментов Стилтьеса, в которой по заданной последовательности матрищ $s_{0}, \ldots, s_{k}, \cdots \in \mathbb{C}_{H}^{m \times m}$ требуется описать множество монотонно возрастающих матриц-функций $(\mathrm{M} \Phi) \sigma: \mathbb{R}_{+} \rightarrow \mathbb{C}_{H}^{m \times m}$ таких, что

$$
s_{j}=\int_{0}^{+\infty} t^{j} d \sigma(t) \quad \forall j \geqslant 0 .
$$

Можем считать, не изменяя значений интегралов, что М $\sigma$ удовлетворяет следующим условиям нормировки: $\sigma(t)$ непрерьвна слева при $t \in(0,+\infty)$ и $\sigma^{\sim}(0)=0$. Множество нормированных решений $\sigma$ проблемы моментов $(1)$ обозначим символом $\mathscr{M}_{\infty}$. С каждой $\sigma \in \mathscr{M}_{\infty}$ свяжем М $\Phi$

$$
s(z)=\int_{0}^{+\infty} \frac{d \sigma(t)}{t-z} .
$$

$\mathrm{M} \Phi s$ определена и голоморфна в $\mathbb{C} \backslash \mathbb{R}_{+}$и называется ассоциированной с проблемой моментов (1). Множество МФ $s$, ассоциированных с проблемой (1), обозначим символом $\mathscr{F} \infty$. Из формулы обрашения Стилтьеса следует, что соответствие, устанавливаемое 
между $\mathscr{F}_{\infty}$ и $\mathscr{M}_{\infty}$ формулой $(2)$, является взаимно однозначным. Поэтому вместо описания множества $\mathscr{M}_{\infty}$ мы можем ограничиться описанием множества $\mathscr{F}_{\infty}$.

В неопределенном случае (см. определение 5) существует бесконечно много решений проблемы моментов (1) и множество ассоциированных $\mathrm{M} \Phi \mathscr{F}_{\infty}$ описьвается в терминах дробно-линейных преобразований. Матрица коэффициентов такого дробно-линейного преобразования назьвается резольвентной матрицей.

Приведем краткое описание основных результатов статьи.

1. Теорема 6 является критерием неопределенности проблемы моментов (1). Этот критерий является новым даже для скалярного случая $(m=1)$.

2. Теорема 8 дает еще один критерий неопределенности проблемы (1). Этот критерий является обобщением на матричный случай классического критерия, принадлежащего Стилтьесу [1]. Он сформулирован в терминах параметров Стилтьеса $M_{j}$ и $L_{j}$. Ранее были известны лишь неявные (рекурсивные) процедуры нахождения этих параметров. Полученные в статье явные формулы (37), связьвающие параметры $M_{j}$ и $L_{j}$ с исходными моментами, являются новьги даже для скалярного случая.

3. Теоремы 9 и 10 показьвают, что элементы резольвентной матрицы неопределенной проблемы моментов (1) являются целыми функциями не вьше первого порядка минимального экспоненциального типа. Особо отметим простое доказательство теоремы 10. Для скалярного случая эти теоремы доказаны, например, в [2].

4. Теорема 12 дает описание в неопределенном случае множества $\mathscr{F} \infty$ в терминах дробно-линейных преобразований.

5. К основным результатам относятся формулы $(16),(27)$ и (29), играющие ключевую роль в этой статье. Эти формулы отражают некоторые фундаментальные свойства матричной проблемы моментов Стилтьеса. Они не имеют аналогов в проблеме моментов Гамбургера.

2. Усеченная проблема моментов Стилтьеса. Вместе с бесконечной проблемой моментов (1) будем рассматривать и усеченные матричные проблемы моментов Стилтьеса. В таких проблемах фиксируется число $k \geqslant 0$ и требуется описать всенормированные монотонно возрастаюшие $\mathrm{M} \Phi \sigma: \mathbb{R}_{+} \rightarrow \mathbb{C}_{H}^{m \times m}$ и матрицы $M \in \mathbb{C}_{\geqslant}^{m \times m}$ такие, что

$$
s_{j}=\int_{0}^{+\infty} t^{j} d \sigma(t), \quad 0 \leqslant j \leqslant k-1, \quad s_{k}=\int_{0}^{+\infty} t^{k} d \sigma(t)+M .
$$

При $k=0$ требуем выполнения только второго условия в (3). Матрища $M$ в (3) позволяет учесть возможный скачок МФ $\sigma$ в бесконечности (см. [3]).

Проблема моментов (3) назьвается $k$-й усеченной проблемой моментов Cтилть$e c a$, а множество ее решений $\sigma$ обозначается символом $\mathscr{M}_{k}$. Ясно, что $\mathscr{M}_{\infty}=\bigcap_{k=0}^{\infty} \mathscr{M}_{k}$. Как и в случае проблемы моментов (1), с каждой $\sigma \in \mathscr{M}_{k}$ свяжем ассоциированную M $\Phi$ вида (2). Множество всех $\mathrm{M} \Phi s$, ассоциированных с проблемой (3), обозначим символом $\mathscr{F}_{k}$.

При $k=2 n, n \geqslant 1$, с усеченной проблемой (3) свяжем следующие блок-матрищы (каждый блок является $m \times m$ матрицей):

$$
K_{1}=\underbrace{\left[\begin{array}{cccc}
s_{0} & s_{1} & \ldots & s_{n} \\
s_{1} & s_{2} & \ldots & s_{n+1} \\
\vdots & \vdots & \ddots & \vdots \\
s_{n} & s_{n+1} & \ldots & s_{2 n}
\end{array}\right]}_{(n+1) m}, \quad L_{1}=\underbrace{\left[\begin{array}{ccc}
I & \ldots & 0 \\
\vdots & \ddots & \vdots \\
0 & \ldots & I \\
0 & \ldots & 0
\end{array}\right]}_{n m}, \quad u_{1}=\underbrace{\left[\begin{array}{c}
0 \\
-s_{0} \\
\vdots \\
-s_{n-1}
\end{array}\right]}_{m}
$$




$$
\begin{aligned}
& v_{1}=\underbrace{\left[\begin{array}{c}
I \\
0 \\
\vdots \\
0
\end{array}\right]}_{m}, \quad T_{1}=\underbrace{\left[\begin{array}{cccc}
0 & \ldots & 0 & 0 \\
I & \ldots & 0 & 0 \\
\vdots & \ddots & \vdots & \vdots \\
0 & \ldots & I & 0
\end{array}\right]}_{(n+1) m}, \quad R_{T_{1}}(z)=\underbrace{\left[\begin{array}{cccc}
I & \ldots & 0 & 0 \\
z I & \ldots & 0 & 0 \\
\vdots & \ddots & \vdots & \vdots \\
z^{n} I & \ldots & z I & I
\end{array}\right]}_{(n+1) m}, \\
& K_{2}=\underbrace{\left[\begin{array}{cccc}
s_{1} & s_{2} & \ldots & s_{n} \\
s_{2} & s_{3} & \ldots & s_{n+1} \\
\vdots & \vdots & \ddots & \vdots \\
s_{n} & s_{n+1} & \ldots & s_{2 n-1}
\end{array}\right]}_{n m}, \quad L_{2}=\underbrace{\left[\begin{array}{ccc}
0 & \ldots & 0 \\
I & \ldots & 0 \\
\vdots & \ddots & \vdots \\
0 & \ldots & I
\end{array}\right]}_{n m}, \quad u_{2}=\underbrace{\left[\begin{array}{c}
-s_{0} \\
-s_{1} \\
\vdots \\
-s_{n-1}
\end{array}\right]}_{m}, \\
& v_{2}=\underbrace{\left[\begin{array}{c}
I \\
0 \\
\vdots \\
0
\end{array}\right]}_{m}, \quad T_{2}=\underbrace{\left[\begin{array}{cccc}
0 & \ldots & 0 & 0 \\
I & \ldots & 0 & 0 \\
\vdots & \ddots & \vdots & \vdots \\
0 & \ldots & I & 0
\end{array}\right]}_{n m}, \quad R_{T_{2}}(z)=\underbrace{\left[\begin{array}{cccc}
I & \ldots & 0 & 0 \\
z I & \ldots & 0 & 0 \\
\vdots & \ddots & \vdots & \vdots \\
z^{n-1} I & \ldots & z I & I
\end{array}\right]}_{n m} .
\end{aligned}
$$

При $k=2 n+1, n \geqslant 0$ с усеченной проблемой моментов (3) свяжем следуюшие блок-матрицы (каждый блок является $m \times m$ матрищей и каждая блок-матрица имеет $(n+1) m$ строк):

$$
\begin{aligned}
& K_{1}=\underbrace{\left[\begin{array}{cccc}
s_{0} & s_{1} & \ldots & s_{n} \\
s_{1} & s_{2} & \ldots & s_{n+1} \\
\vdots & \vdots & \ddots & \vdots \\
s_{n} & s_{n+1} & \ldots & s_{2 n}
\end{array}\right]}_{(n+1) m}, \quad L_{1}=\underbrace{\left[\begin{array}{cccc}
I & \ldots & 0 & 0 \\
\vdots & \ddots & \vdots & \vdots \\
0 & \ldots & I & 0 \\
0 & \ldots & 0 & I
\end{array}\right]}_{(n+1) m}, \quad u_{1}=\underbrace{\left[\begin{array}{c}
0 \\
-s_{0} \\
\vdots \\
-s_{n-1}
\end{array}\right]}_{m}, \\
& v_{1}=\underbrace{\left[\begin{array}{c}
I \\
0 \\
\vdots \\
0
\end{array}\right]}_{m}, \quad T_{1}=\underbrace{\left[\begin{array}{cccc}
0 & \ldots & 0 & 0 \\
I & \ldots & 0 & 0 \\
\vdots & \ddots & \vdots & \vdots \\
0 & \ldots & I & 0
\end{array}\right]}_{(n+1) m}, \quad R_{T_{1}}(z)=\underbrace{\left[\begin{array}{cccc}
I & \ldots & 0 & 0 \\
z I & \ldots & 0 & 0 \\
\vdots & \ddots & \vdots & \vdots \\
z^{n} I & \ldots & z I & I
\end{array}\right]}_{(n+1) m}, \\
& K_{2}=\underbrace{\left[\begin{array}{cccc}
s_{1} & s_{2} & \ldots & s_{n+1} \\
s_{2} & s_{3} & \ldots & s_{n+2} \\
\vdots & \vdots & \ddots & \vdots \\
s_{n+1} & s_{n+2} & \ldots & s_{2 n+1}
\end{array}\right]}_{(n+1) m}, \quad L_{2}=\underbrace{\left[\begin{array}{cccc}
0 & \ldots & 0 & 0 \\
I & \ldots & 0 & 0 \\
\vdots & \ddots & \vdots & \vdots \\
0 & \ldots & I & 0
\end{array}\right]}_{(n+1) m}, \quad v_{2}=\underbrace{\left[\begin{array}{c}
I \\
0 \\
\vdots \\
0
\end{array}\right]}_{m}, \\
& u_{2}=\underbrace{\left[\begin{array}{c}
-s_{0} \\
-s_{1} \\
\vdots \\
-s_{n}
\end{array}\right]}_{m}, \quad T_{2}=\underbrace{\left[\begin{array}{cccc}
0 & \ldots & 0 & 0 \\
I & \ldots & 0 & 0 \\
\vdots & \ddots & \vdots & \vdots \\
0 & \ldots & I & 0
\end{array}\right]}_{(n+1) m}, \quad R_{T_{2}}(z)=\underbrace{\left[\begin{array}{cccc}
I & \ldots & 0 & 0 \\
z I & \ldots & 0 & 0 \\
\vdots & \ddots & \vdots & \vdots \\
z^{n} I & \ldots & z I & I
\end{array}\right]}_{(n+1) m} .
\end{aligned}
$$

Непосредственно проверяем, что определенные выше блок-матрицы при $k=2 n, n \geqslant 1$ и при $k=2 n+1, n \geqslant 0$ удовлетворяют основному тожсдеству

$$
L_{2} K_{2}-K_{1} L_{1}=v_{1} u_{2}^{*} \text {. }
$$


Кроме того, имеют место тождества

$$
\begin{gathered}
T_{1}=L_{2} L_{1}^{*}, \quad T_{2}=L_{1}^{*} L_{2}, \quad u_{1}=L_{2} u_{2}, \quad v_{2}=L_{1}^{*} v_{1}, \\
R_{T_{1}}(z)=\left(I-z T_{1}\right)^{-1}, \quad R_{T_{2}}(z)=\left(I-z T_{2}\right)^{-1}, \quad T_{1} L_{2}=L_{2} T_{2}, \\
T_{2} L_{1}^{*}=L_{1}^{*} T_{1}, \quad R_{T_{1}}(z) L_{2}=L_{2} R_{T_{2}}(z), \quad R_{T_{2}}(z) L_{1}^{*}=L_{1}^{*} R_{T_{1}}(z) .
\end{gathered}
$$

ОПРЕДЕЛЕниЕ 1. Усеченная $k$-я проблема моментов Стилтьеса (3) назьвается неопределенной, если выполнены условия $K_{1}>0, K_{2}>0$.

Всюду в этой статье будем предполагать, что все усеченные проблемы моментов (3) являются неопределенными.

Рассмотрим две полиномиальные $М \Phi$

$$
\begin{aligned}
& U_{1}(z)=\left[\begin{array}{cc}
I+z v_{2}^{*} R_{T_{2}^{*}}(z) K_{2}^{-1} u_{2} & -z v_{1}^{*} R_{T_{1}^{*}}(z) K_{1}^{-1} v_{1} \\
u_{2}^{*} R_{T_{2}^{*}}(z) K_{2}^{-1} u_{2} & I-z u_{1}^{*} R_{T_{1}^{*}}(z) K_{1}^{-1} v_{1}
\end{array}\right], \\
& U_{2}(z)=\left[\begin{array}{cc}
I+z v_{2}^{*} R_{T_{2}^{*}}(z) K_{2}^{-1} u_{2} & -v_{1}^{*} R_{T_{1}^{*}}(z) K_{1}^{-1} v_{1} \\
z u_{2}^{*} R_{T_{2}^{*}}(z) K_{2}^{-1} u_{2} & I-z u_{1}^{*} R_{T_{1}^{*}}(z) K_{1}^{-1} v_{1}
\end{array}\right] .
\end{aligned}
$$

Здесь и в дальнейшем $R_{T_{r}^{*}}(z)=\left(I-z T_{r}^{*}\right)^{-1}$ при $r=1,2$.

ОПРЕДЕЛЕниЕ 2. Матрища-функция $U_{1}: \mathbb{C} \rightarrow \mathbb{C}^{2 m \times 2 m}$ назьвается резольвентной матрицей проблемы моментов Стилтьеса (3).

Введем разбиение резольвентной матрицы $U_{1}$ на четыре $m \times m$ блока

$$
U_{1}(z)=\left[\begin{array}{ll}
\alpha(z) & \beta(z) \\
\gamma(z) & \delta(z)
\end{array}\right] \quad \forall z \in \mathbb{C}
$$

Можно доказать, что $\mathrm{M} \Phi \alpha, \beta, \gamma, \delta$ невырождены $\forall z \in \mathbb{C} \backslash \mathbb{R}_{+} \cdot$ Пусть

$$
J=\left[\begin{array}{cc}
0_{m \times m} & -i I_{m \times m} \\
i I_{m \times m} & 0_{m \times m}
\end{array}\right]
$$

Непосредственные вычисления с использованием (4) и (5) приводят к следующим выражениям для $J$-форм $\mathrm{M} \Phi U_{1}$ и $U_{2}$ :

$$
J-U_{r}(z) J U_{r}^{*}(\lambda)=i(z-\bar{\lambda})\left[\begin{array}{c}
v_{r}^{*} \\
u_{r}^{*}
\end{array}\right] R_{T_{r}^{*}}(z) K_{r}^{-1} R_{T_{r}^{*}}^{*}(\lambda)\left[v_{r}, u_{r}\right], \quad r=1,2 .
$$

Умножим последнее равенство справа на $J$ и подставим в него $\bar{z}$ вместо $\lambda$. Учитьвая равенство $J^{2}=I$, приходим к принципу симметрии

$$
U_{r}^{-1}(z)=J U_{r}^{*}(\bar{z}) J, \quad r=1,2 .
$$

Подставим в (9) $\bar{z}$ вместо $z$ и $\lambda$ и умножим (9) слева и справа на $J$. Из принципа симметрии и очевидного равенства $R_{T_{r}^{*}}^{*}(\bar{z})=R_{T_{r}}(z)$ следует, что

$$
J-U_{r}^{-1^{*}}(z) J U_{r}^{-1}(z)=i(\bar{z}-z) J\left[\begin{array}{c}
v_{r}^{*} \\
u_{r}^{*}
\end{array}\right] R_{T_{r}}^{*}(z) K_{r}^{-1} R_{T_{r}}(z)\left[\begin{array}{ll}
v_{r} & \left.u_{r}\right] J .
\end{array}\right.
$$


Непосредственные вычисления с использованием (4), (5) и (8) приводят к следующему выражению для $J_{\pi \text {-формы }} \mathrm{M} \Phi U_{1}$ :

$$
\begin{aligned}
& U_{1}(z) J_{\pi} U_{1}^{*}(z)-J_{\pi}=\left[\begin{array}{cc}
\alpha(z) \beta^{*}(z)+\beta(z) \alpha^{*}(z) & -I+\alpha(z) \delta^{*}(z)+\beta(z) \gamma^{*}(z) \\
-I+\gamma(z) \beta^{*}(z)+\delta(z) \alpha^{*}(z) & \gamma(z) \delta^{*}(z)+\delta(z) \gamma^{*}(z)
\end{array}\right] \\
& =-(z+\bar{z})\left[\begin{array}{c}
v_{1}^{*} \\
u_{1}^{*}
\end{array}\right] R_{T_{1}^{*}}(z) K_{1}^{-1} R_{T_{1}^{*}}^{*}(z)\left[v_{1}, u_{1}\right] \\
& +2\left[\begin{array}{c}
z v_{2}^{*} \\
u_{2}^{*}
\end{array}\right] R_{T_{2}^{*}}(z) K_{2}^{-1} R_{T_{2}^{*}}^{*}(z)\left[\bar{z} v_{2}, u_{2}\right], \\
& J_{\pi}=\left[\begin{array}{ll}
0_{m \times m} & I_{m \times m} \\
I_{m \times m} & 0_{m \times m}
\end{array}\right] \text {. }
\end{aligned}
$$

ТЕОРемА 1. Пусть МФ $s$ является ассоциированной с неопределенной проблемой моментов (3), а $\mathrm{M} \Phi U_{1}$ и $U_{2}$ определены формулами (6) $и$ (7). Тогда $\forall z \in \mathbb{C}_{ \pm}$ выполнены основные матричные неравенства (ОМН) В. П. Потапова

$$
\left[I \bar{z}^{r-1} s^{*}(z)\right] \frac{U_{r}^{-1^{*}}(z) J U_{r}^{-1}(z)}{i(\bar{z}-z)}\left[\begin{array}{c}
I \\
z^{r-1} s(z)
\end{array}\right] \geqslant 0, \quad z \in \mathbb{C}_{ \pm}, \quad r=1,2 .
$$

Наоборот, пусть для некоторой голоморфной в $\mathbb{C}_{+} \mathrm{M} \Phi$ s неравенства $(12)$ выполняются хотя бъ $\forall z \in \mathbb{C}_{+}$. Тогда s допускает представление (2) и $\sigma \in \mathscr{M}_{k}$, т.е. $s \in \mathscr{F}_{k}$.

Доказательство этой теоремы имеется в [4].

ОПРЕДЕлЕниЕ 3 . . Пара $m \times m \mathrm{M} \Phi \operatorname{col}[p(z) q(z)]$, мероморфных в $\mathbb{C} \backslash \mathbb{R}_{+}$, назьвается стилтьесовской, если для нее существует дискретное в $\mathbb{C} \backslash \mathbb{R}_{+}$множество точек $\mathscr{D}_{p q}$ такое, что

1) $p^{*}(z) p(z)+q^{*}(z) q(z)>0, z \in \mathbb{C} \backslash\left\{\mathbb{R}_{+} \cup \mathscr{D}_{p q}\right\}$

2) $\left[p^{*}(z), q^{*}(z)\right] \frac{J}{i(\bar{z}-z)}\left[\begin{array}{l}p(z) \\ q(z)\end{array}\right] \geqslant 0, z \in \mathbb{C} \backslash\left\{\mathbb{R} \cup \mathscr{D}_{p q}\right\}$;

3) $\left[p^{*}(z), q^{*}(z)\right] J_{\pi}\left[\begin{array}{l}p(z) \\ q(z)\end{array}\right] \geqslant 0, z \in\{z \in \mathbb{C}: \operatorname{Re} z<0\} \backslash \mathscr{D}_{p q}$.

На множестве стилтьесовских пар введем отношение эквивалентности: пары $\operatorname{col}\left[p_{1}(z) q_{1}(z)\right]$ и $\operatorname{col}\left[p_{2}(z) q_{2}(z)\right]$ назьваются әквивалентнылми, если существует $\mathrm{M} \Phi$ $Q(z)$ такая, что в $\mathbb{C} \backslash \mathbb{R}_{+}$мероморфны обе $\mathrm{M} \Phi Q(z),(Q(z))^{-1}$ и $p_{1}(z)=p_{2}(z) Q(z)$, $q_{1}(z)=q_{2}(z) Q(z)$. Множество классов эквивалентности стилтьесовских пар обозначим через $\mathscr{S}_{\infty}$.

Пусть $A, B \in \mathbb{C}^{m \times m}$ и матрища $B$ невырождена. Тогда $\frac{A}{B}:=A \cdot B^{-1}$.

ТЕОрема 2. Пусть проблема моментов (3) является неопределенной и резольвентная матрича $U_{1}$ разбита на четыре $m \times m$ блока $\alpha, \beta, \gamma, \delta$ (см. (8)).

Тогда формула

$$
s(z)=\frac{\gamma(z) p(z)+\delta(z) q(z)}{\alpha(z) p(z)+\beta(z) q(z)}
$$

устанавливает биективное соответствие между $\mathscr{F}_{k}$ и $\mathscr{S}_{\infty}$.

Доказательство использует систему ОМН (12) и проводится по аналогии с доказательством аналогичного утверждения в [5]. 
3. Экстремальные решения и интервалы Вейля. Подставляя в (13) стилтьесовские пары $\operatorname{col}\left[\begin{array}{ll}I & 0]\end{array}\right.$ и $\operatorname{col}[0 I]$, получим экстремальные $\mathrm{M} \Phi$

$$
s_{\mu}(z)=\frac{\gamma(z)}{\alpha(z)} \in \mathscr{F}_{k}, \quad s_{M}(z)=\frac{\delta(z)}{\beta(z)} \in \mathscr{F}_{k} .
$$

ТЕОРемА 3. Экстремальные МФ допускают представления

$$
s_{\mu}(z)=u_{2}^{*}\left(K_{2}-z L_{1}^{*} K_{1} L_{1}\right)^{-1} u_{2}, \quad s_{M}(z)=\left\{v_{1}^{*}\left(L_{2} K_{2} L_{2}^{*}-z K_{1}\right)^{-1} v_{1}\right\}^{-1}
$$

ДокАЗАТЕЛЬСТво. Из равенства

$$
s_{\mu}(z)=u_{2}^{*} K_{2}^{-1 / 2}\left(I-z K_{2}^{-1 / 2} L_{1}^{*} K_{1} L_{1} K_{2}^{-1 / 2}\right)^{-1} K_{2}^{-1 / 2} u_{2}
$$

и свойств резольвенты неотрицательной эрмитовой матрицы следует, что $s_{\mu}(z)$ из $(15)$ определена и голоморфна в $\mathbb{C} \backslash \mathbb{R}_{+}$. Далее имеем

$$
\begin{aligned}
u_{2}^{*}\left(K_{2}-z\right. & \left.L_{1}^{*} K_{1} L_{1}\right)^{-1} u_{2} \cdot \alpha(z) \\
& =u_{2}^{*}\left(K_{2}-z L_{1}^{*} K_{1} L_{1}\right)^{-1} u_{2}\left\{I+z v_{2}^{*} R_{T_{2}^{*}}(z) K_{2}^{-1} u_{2}\right\} \\
& =u_{2}^{*}\left(K_{2}-z L_{1}^{*} K_{1} L_{1}\right)^{-1}\left\{K_{2}\left(I-z T_{2}^{*}\right)+z u_{2} v_{2}^{*}\right\} R_{T_{2}^{*}}(z) K_{2}^{-1} u_{2} \\
& =u_{2}^{*}\left(K_{2}-z L_{1}^{*} K_{1} L_{1}\right)^{-1}\left\{K_{2}\left(I-z T_{2}^{*}\right)+z u_{2} v_{1}^{*} L_{1}\right\} R_{T_{2}^{*}}(z) K_{2}^{-1} u_{2} \\
& =u_{2}^{*}\left(K_{2}-z L_{1}^{*} K_{1} L_{1}\right)^{-1}\left\{K_{2}\left(I-z T_{2}^{*}\right)+z\left(K_{2} L_{2}^{*}-L_{1}^{*} K_{1}\right) L_{1}\right\} R_{T_{2}^{*}}(z) K_{2}^{-1} u_{2} \\
& =u_{2}^{*}\left(K_{2}-z L_{1}^{*} K_{1} L_{1}\right)^{-1}\left\{K_{2}-z L_{1}^{*} K_{1} L_{1}\right\} R_{T_{2}^{*}}(z) K_{2}^{-1} u_{2} \\
& =u_{2}^{*} R_{T_{2}^{*}}(z) K_{2}^{-1} u_{2}=\gamma(z) .
\end{aligned}
$$

В этой цепочке равенств первое вытекает из (8) и (6), третье - из (5), четвертое - из (4), пятое - из (5), седьмое - из (8) и (6). Таким образом, $s_{\mu}(z)=\gamma(z) \alpha^{-1}(z)=u_{2}^{*}\left\{K_{2}-\right.$ $\left.z L_{1}^{*} K_{1} L_{1}\right\}^{-1} u_{2}$. Первое из равенств (15) доказано. Второе из равенств (15) доказьвается аналогичным образом.

ТЕОремА 4. Пусть дана неопределенная проблема моментов (3) и $\mathscr{F}_{k}$ обозначает множество ассоциированных МФ. И пусть, далее, $s_{\mu} u s_{M}$ - әкстремальные ассоциированные МФ. Тогда

1) $\mathrm{M} \Phi\left\{s_{M}(z)-s_{\mu}(z)\right\}^{-1}$ голоморфна в $\mathbb{C} \backslash \mathbb{R}_{+} u$

$$
\left\{s_{M}(z)-s_{\mu}(z)\right\}^{-1}=-z v_{1}^{*} R_{T_{1}^{*}}(z) K_{1}^{-1} R_{T_{1}}(z) v_{1}+z^{2} v_{2}^{*} R_{T_{2}^{*}}(z) K_{2}^{-1} R_{T_{2}}(z) v_{2}
$$

2) $s_{M}(x)>s_{\mu}(x) \forall x \in \mathbb{R}_{-}$;

3) $s_{\mu}(x) \leqslant s(x) \leqslant s_{M}(x) \forall s \in \mathscr{F}_{k}, \forall x \in \mathbb{R}_{-}$. 
ДокАЗАТЕЛЬСтво. 1) Пусть $x \in \mathbb{R}_{-}$. Подставим $x$ в формулу (9) вместо $z$ и $\lambda$. Получим $J-U_{1}(x) J U_{1}^{*}(x)=0$. Но тогда (см. [6]) и $J-U_{1}^{*}(x) J U_{1}(x)=0$. Отсюда и из (8) следует, что

$$
\left[\begin{array}{cc}
-\alpha^{*}(x) \gamma(x)+\gamma^{*}(x) \alpha(x) & -\alpha^{*}(x) \delta(x)+\gamma^{*}(x) \beta \\
-\beta^{*}(x) \gamma(x)+\delta^{*}(x) \alpha(x) & -\beta^{*}(x) \delta(x)+\delta^{*}(x) \beta(x)
\end{array}\right]=\left[\begin{array}{cc}
0 & -I \\
I & 0
\end{array}\right] .
$$

Таким образом, $-\beta^{*}(x) \gamma(x)+\delta^{*}(x) \alpha(x)=I$. В силу (15) $s_{M}(x)=s_{M}^{*}(x)$. Поэтому

$$
\begin{aligned}
s_{M}(x)-s_{\mu}(x) & =\beta^{-1^{*}}(x) \delta^{*}(x)-\gamma(x) \alpha^{-1}(x) \\
& =\beta^{-1^{*}}(x)\left\{\delta^{*}(x) \alpha(x)-\beta^{*}(x) \gamma(x)\right\} \alpha^{-1}(x)=\beta^{-1^{*}}(x) \alpha^{-1}(x) .
\end{aligned}
$$

Как уже отмечалось, полиномиальные $\mathrm{M} \Phi \alpha(z)$ и $\beta(z)$ невырождены в $\mathbb{C} \backslash \mathbb{R}_{+}$. Поэтому рациональные $\mathrm{M} \Phi \alpha^{-1}(z)$ и $\beta^{-1^{*}}(\bar{z})$ голоморфны в $\mathbb{C} \backslash \mathbb{R}_{+}$. Из $(20)$ следует, что и $\mathrm{M} \Phi$ $\left\{s_{M}(z)-s_{\mu}(z)\right\}^{-1}$ голоморфна в $\mathbb{C} \backslash \mathbb{R}_{+}$. Далее

$$
\begin{aligned}
\left\{s_{M}(x)-s_{\mu}(x)\right\}^{-1}= & \alpha(x) \beta^{*}(x)=-x\left\{I+x v_{2}^{*} R_{T_{2}^{*}}(x) K_{2}^{-1} u_{2}\right\} \cdot\left\{v_{1}^{*} K_{1}^{-1} R_{T_{1}}(x) v_{1}\right\} \\
= & -x\left\{v_{1}^{*} K_{1}^{-1} R_{T_{1}}(x) v_{1}\right. \\
& \left.+x v_{2}^{*} R_{T_{2}^{*}}(x) K_{2}^{-1}\left(-L_{1}^{*} K_{1}+K_{2} L_{2}^{*}\right) K_{1}^{-1} R_{T_{1}}(x) v_{1}\right\} \\
= & -x\left\{v_{1}^{*} K_{1}^{-1} R_{T_{1}}(x) v_{1}-x v_{2}^{*} R_{T_{2}^{*}}(x) K_{2}^{-1} L_{1}^{*} R_{T_{1}}(x) v_{1}\right. \\
& \left.+x v_{2}^{*} R_{T_{2}^{*}}(x) L_{2}^{*} K_{1}^{-1} R_{T_{1}}(x) v_{1}\right\} \\
= & -x\left\{v_{1}^{*} K_{1}^{-1} R_{T_{1}}(x) v_{1}-x v_{2}^{*} R_{T_{2}^{*}}(x) K_{2}^{-1} L_{1}^{*} R_{T_{1}}(x) v_{1}\right. \\
& \left.+x v_{1}^{*} L_{1} L_{2}^{*} R_{T_{1}^{*}}(x) K_{1}^{-1} R_{T_{1}}(x) v_{1}\right\} \\
=- & x\left\{v_{1}^{*} K_{1}^{-1} R_{T_{1}}(x) v_{1}-x v_{2}^{*} R_{T_{2}^{*}}(x) K_{2}^{-1} L_{1}^{*} R_{T_{1}}(x) v_{1}\right. \\
& \left.+x v_{1}^{*} T_{1}^{*} R_{T_{1}^{*}}(x) K_{1}^{-1} R_{T_{1}}(x) v_{1}\right\} \\
= & -x\left\{v_{1}^{*}\left[I-x T_{1}^{*}+x T_{1}^{*}\right] R_{T_{1}^{*}}(x) K_{1}^{-1} R_{T_{1}}(x) v_{1}\right. \\
& \left.-x v_{2}^{*} R_{T_{2}^{*}}(x) K_{2}^{-1} L_{1}^{*} R_{T_{1}}(x) v_{1}\right\} \\
=- & -x\left\{v_{1}^{*} R_{T_{1}^{*}}(x) K_{1}^{-1} R_{T_{1}}(x) v_{1}-x v_{2}^{*} R_{T_{2}^{*}}(x) K_{2}^{-1} L_{1}^{*} R_{T_{1}}(x) v_{1}\right\} \\
= & -x\left\{v_{1}^{*} R_{T_{1}^{*}}(x) K_{1}^{-1} R_{T_{1}}(x) v_{1}-x v_{2}^{*} R_{T_{2}^{*}}(x) K_{2}^{-1} R_{T_{2}}(x) v_{2}\right\} .
\end{aligned}
$$

В этой цепочке равенств второе равенство следует из (8) и (6), третье - из (4), пятое, шестое и девятое - из (5). Таким образом, при $x<0$

$$
\left\{s_{M}(x)-s_{\mu}(x)\right\}^{-1}=-x\left\{v_{1}^{*} R_{T_{1}^{*}}(x) K_{1}^{-1} R_{T_{1}}(x) v_{1}-x v_{2}^{*} R_{T_{2}^{*}}(x) K_{2}^{-1} R_{T_{2}}(x) v_{2}\right\} .
$$

Отсюда в силу аналитичности следует (16).

2) Это утверждение непосредственно следует из (16).

3) Пусть М $\Phi s \in \mathscr{F}_{k}$. Тогда она допускает представление (13) с стилтьесовской парой $\operatorname{col}[p q]$. Пусть $x \in \mathbb{R}_{-} \backslash \mathscr{D}_{p q}$. Рассмотрим разность

$$
\begin{aligned}
s_{M}(x)-s(x)= & \delta(x) \beta^{-1}(x)-\{\gamma(x) p(x)+\delta(x) q(x)\} \cdot\{\alpha(x) p(x)+\beta(x) q(x)\}^{-1} \\
= & \beta^{-1^{*}}(x) \delta^{*}(x)-\{\gamma(x) p(x)+\delta(x) q(x)\} \cdot\{\alpha(x) p(x)+\beta(x) q(x)\}^{-1} \\
= & \beta^{-1^{*}}(x)\left\{\left[\delta^{*}(x) \alpha(x)-\beta^{*}(x) \gamma(x)\right] p(x)\right. \\
& \left.+\left[\delta^{*}(x) \beta(x)-\beta^{*}(x) \delta(x)\right] q(x)\right\}\{\alpha(x) p(x)+\beta(x) q(x)\}^{-1} \\
= & \beta^{-1^{*}}(x) p(x)\{\alpha(x) p(x)+\beta(x) q(x)\}^{-1} .
\end{aligned}
$$


В этой цепочке равенств второе равенство следует из (14) и (15), а последнее - из (19). Окончательно

$$
s_{M}(x)-s(x)=\beta^{-1^{*}}(x) p(x)\{\alpha(x) p(x)+\beta(x) q(x)\}^{-1} .
$$

Таким образом, разность $s_{M}(x)-s(x)$ представлена в виде дробно-линейного преобразования, матрица которого

$$
\widetilde{U}(x)=\left[\begin{array}{cc}
\alpha(x) & \beta(x) \\
\beta^{-1^{*}}(x) & 0
\end{array}\right] .
$$

Очевидно, что ее $J_{\pi}$-форма имеет вид

$$
\widetilde{U}^{*}(x) J_{\pi} \widetilde{U}(x)-J_{\pi}=\left[\begin{array}{cc}
\beta^{-1}(x)\left[\beta(x) \alpha^{*}(x)+\alpha(x) \beta^{*}(x)\right] \beta^{-1^{*}}(x) & 0 \\
0 & 0
\end{array}\right] .
$$

Из (11) следует, что $U_{1}(x) J_{\pi} U_{1}^{*}(x)-J_{\pi} \geqslant 0$. Но тогда, снова из $(11), \beta(x) \alpha^{*}(x)+$ $\alpha(x) \beta^{*}(x) \geqslant 0$. Поэтому $\widetilde{U}^{*}(x) J_{\pi} \widetilde{U}(x) \geqslant J_{\pi}$. С учетом этого неравенства получаем

$$
\begin{aligned}
& {\left[p^{*}(x) \alpha^{*}(x)+q^{*}(x) \beta^{*}(x), p^{*}(x) \beta^{-1}(x)\right] J_{\pi}\left[\begin{array}{c}
\alpha(x) p(x)+\beta(x) q(x) \\
\beta^{-1^{*}}(x) p(x)
\end{array}\right]} \\
& \quad=\left[p^{*}(x), q^{*}(x)\right]\left[\begin{array}{cc}
\alpha^{*}(x) & \beta^{-1}(x) \\
\beta^{*}(x) & 0
\end{array}\right] J_{\pi}\left[\begin{array}{cc}
\alpha(x) & \beta(x) \\
\beta^{-1^{*}}(x) & 0
\end{array}\right]\left[\begin{array}{c}
p(x) \\
q(x)
\end{array}\right] \\
& \quad=\left[p^{*}(x), q^{*}(x)\right] \widetilde{U}^{*}(x) J_{\pi} \widetilde{U}(x)\left[\begin{array}{l}
p(x) \\
q(x)
\end{array}\right] \geqslant\left[p^{*}(x), q^{*}(x)\right] J_{\pi}\left[\begin{array}{l}
p(x) \\
q(x)
\end{array}\right] \geqslant 0 .
\end{aligned}
$$

Последнее неравенство вытекает из определения 3. Таким образом,

$$
\left[p^{*}(x) \alpha^{*}(x)+q^{*}(x) \beta^{*}(x), p^{*}(x) \beta^{-1}(x)\right] J_{\pi}\left[\begin{array}{c}
\alpha(x) p(x)+\beta(x) q(x) \\
\beta^{-1^{*}}(x) p(x)
\end{array}\right] \geqslant 0 .
$$

Умножим это неравенство справа на матрицу $\{\alpha(x) p(x)+\beta(x) q(x)\}^{-1}$, а слева на сопряженную матрицу. Получим

$$
\left[I, s_{M}^{*}(x)-s^{*}(x)\right] J_{\pi}\left[\begin{array}{c}
I \\
s_{M}(x)-s(x)
\end{array}\right] \geqslant 0 .
$$

Или, учитьвая вид матрицы $J_{\pi}$,

$$
s_{M}^{*}(x)-s^{*}(x)+s_{M}(x)-s(x) \geqslant 0 .
$$

М $\Phi s_{M}$ и $s$ принадлежат классу $\mathscr{F}_{k}$ и, следовательно, допускают представления вида $(2)$, откуда следует, что $s_{M}(x)$ и $s(x)$ эрмитовы. Но тогда из последнего неравенства следует, что $s_{M}(x) \geqslant s(x)$. Это неравенство продолжается в точки $x \in \mathbb{R}_{-} \cap \mathscr{D}_{p q}$ по непрерьвности. Неравенство $s_{\mu}(x) \leqslant s(x)$ доказьвается аналогичным образом. 
ОПРЕДЕЛЕНИЕ 4. Пусть матрицы $A, B \in \mathbb{C}_{>}^{m \times m}$ и выполнено условие $A \leqslant B . M a m$ ричным интервалом называется

$$
[A, B]=\left\{\kappa \in \mathbb{C}_{H}^{m \times m}: A \leqslant \kappa \leqslant B\right\} .
$$

Матричный интервал $[A, B]$ назьвается невырожденным, если $A<B$.

Пусть некоторая точка $x_{0} \in \mathbb{R}_{-}$. Матричньй интервал

$$
\mathscr{I}\left(x_{0}\right)=\left[s_{\mu}\left(x_{0}\right), s_{M}\left(x_{0}\right)\right]
$$

назьвается интервалом Вейля.

Неравенство (18) означает, что $\left\{s\left(x_{0}\right): s \in \mathscr{F}_{k}\right\} \subset \mathscr{I}\left(x_{0}\right)$. Можно доказать, что $\left\{s\left(x_{0}\right): s \in \mathscr{F}_{k}\right\}=\mathscr{I}\left(x_{0}\right)$.

4. Предельные интервалы Вейля. Теперь мы исследуем свойства введенных выше объектов при изменяющихся $k$. Для этого в обозначения объектов введем верхний индекс $(k)$, которьй будет указьвать, что соответствуюшие интервалы Вейля, резольвентные матрищы и т.д. связаны с $k$-й усеченной проблемой моментов (3).

Пусть даны целые числа $k_{2}>k_{1}>0$. Из очевидного включения $\mathscr{F}_{k_{2}} \subset \mathscr{F}_{k_{1}}$ следует, что

$$
\mathscr{I}^{\left(k_{2}\right)}\left(x_{0}\right) \subset \mathscr{I}^{\left(k_{1}\right)}\left(x_{0}\right) \quad \forall x_{0} \in \mathbb{R}_{-} .
$$

ТЕОремА 5. Пусть при любом $k>0$ проблема моментов (3) является неопределенной и $\mathscr{F}_{k}$ обозначает мнохсество ассоииированных МФ. И пусть, далее, $s_{\mu}^{(k)} u s_{M}^{(k)}$ - соответствующие әкстремальные МФ. Тогда

1) существуют предель

$$
\lim _{k \rightarrow+\infty} s_{M}^{(k)}(x)>0, \quad \lim _{k \rightarrow+\infty} s_{\mu}^{(k)}(x)>0 \quad \forall x \in \mathbb{R}_{-}
$$

2) существуют равномерные на компактах из $\mathbb{C} \backslash \mathbb{R}_{+}$предель

$$
s_{M}^{(\infty)}(z):=\lim _{k \rightarrow+\infty} s_{M}^{(k)}(z), \quad s_{\mu}^{(\infty)}(z):=\lim _{k \rightarrow+\infty} s_{\mu}^{(k)}(z)
$$

3) $s_{M}^{(\infty)}, s_{\mu}^{(\infty)} \in \mathscr{F}_{\infty}$;

4) $s_{\mu}^{(\infty)}(x) \leqslant s_{M}^{(\infty)}(x) \forall x \in \mathbb{R}_{-}$;

5) $s_{\mu}^{(\infty)}(x) \leqslant s(x) \leqslant s_{M}^{(\infty)}(x) \forall x \in \mathbb{R}_{-}, \forall s \in \mathscr{F}_{\infty}$.

ДокАЗАтЕльство. 1) Из включения $\mathscr{I}^{\left(k_{2}\right)}(x) \subset \mathscr{I}^{\left(k_{1}\right)}(x), \forall x \in \mathbb{R}_{-}, k_{2}>k_{1}>0$ (см. (21)) вытекает неравенство $s_{M}^{\left(k_{2}\right)}(x) \leqslant s_{M}^{\left(k_{1}\right)}(x)$. Поэтому в каждой точке $x \in \mathbb{R}_{-}$ последовательность эрмитовых матриц $\left\{s_{M}^{(k)}(x)\right\}_{k=1}^{\infty}$ монотонно убывает и ограничена снизу строго положительной матрицей $s_{\mu}^{(1)}(x)$. Отсюда следует, что существует предел $\lim _{k \rightarrow+\infty} s_{M}^{(k)}(x)>0 \forall x \in \mathbb{R}_{-}$. Существование второго предела в (22) доказьвается аналогичньм образом.

2) Покажем, что последовательность $\mathrm{M} \Phi\left\{s_{M}^{(k)}\right\}_{k=1}^{\infty}$ равномерно ограничена на компактах из $\mathbb{C} \backslash \mathbb{R}_{+}$. При фиксированном $x \in \mathbb{R}_{-}$сходящаяся матричная последовательность $\left\{s_{M}^{(k)}(x)\right\}_{k=1}^{\infty}$ ограничена. Отсюда и из представления $\mathrm{M} \Phi s_{M}^{(k)}$ в виде $(2)$ последовательно получаем ограниченность семейства $\left\{s_{M}^{(k)}\right\}_{k=1}^{\infty}$ на компактах из левой, верхней 
и нижней полуплоскостей (см. [7, с. 32]). Но тогда рассматриваемое семейство ограничено и на произвольном компакте из $\mathbb{C} \backslash \mathbb{R}_{+}$.

Пусть теперь $K$ - произвольный компакт из $\mathbb{C} \backslash \mathbb{R}_{+}$. Обозначим через $\Omega$ односвязную открытую область с гладкой гранищей такую, что

1) $K \subset \Omega$;

2) замыкание $\Omega$ является компактом в $\mathbb{C} \backslash \mathbb{R}_{+}$;

$3)$ область $\Omega$ содержит некоторьй интервал $(a, b) \subset \mathbb{R}_{\_}$.

Последовательность М $\left\{s_{M}^{(k)}\right\}_{k=1}^{\infty}$ равномерно ограничена в замыкании области $\Omega$, а значит, и в самой области $\Omega$. Последовательность матриц $\left\{s_{M}^{(k)}(x)\right\}_{k=1}^{\infty}$ сходится $\forall x \in$ $(a, b)$. По теореме Витали последовательность $\mathrm{M} \Phi\left\{s_{M}^{(k)}\right\}_{k=1}^{\infty}$ равномерно сходится при $z \in K$. Мы доказали существование первого из пределов в (23). Аналогичньм образом доказьвается существование второго предела в (23).

3) Пусть зафиксировано целое число $k>0$ и пусть $l>k$. Тогда $s_{M}^{(l)} \in \mathscr{F}_{k}$ и, следовательно, вьполнена система ОМН Потапова

$$
\left[I \bar{z}^{r-1} s_{M}^{(l)^{*}}(z)\right] \frac{U_{r}^{(k)^{-1^{*}}}(z) J U_{r}^{(k)^{-1}}(z)}{i(\bar{z}-z)}\left[\begin{array}{c}
I \\
z^{r-1} s_{M}^{(l)}(z)
\end{array}\right] \geqslant 0, \quad z \in \mathbb{C}_{ \pm}, \quad r=1,2
$$

Перейдем в этих неравенствах к пределу $l \rightarrow \infty$. Получим, что $s_{M}^{(\infty)} \in \mathscr{F}_{k}$. В силу произвольности $k$ отсюда следует, что $s_{M}^{(\infty)} \in \mathscr{F}_{\infty}$. Утверждение относительно $s_{\mu}^{(\infty)}$ доказьвается аналогичньм образом.

4) В неравенстве $s_{\mu}^{(k)}(x)<s_{M}^{(k)}(x) \forall x \in \mathbb{R}_{-}$перейдем к пределу $k \rightarrow \infty$.

$5)$ Пусть задана некоторая $\mathrm{M} \Phi s \in \mathscr{F}_{\infty}$. Ясно, что $s \in \mathscr{F}_{k} \forall k>0$. По теореме 4 имеют место неравенства $s_{\mu}^{(k)}(x) \leqslant s(x) \leqslant s_{M}^{(k)}(x)$. Переходя к пределу $k \rightarrow \infty$, получим (26).

Пусть некоторая точка $x \in \mathbb{R}_{-}$. Матричньй интервал

$$
\mathscr{I}^{(\infty)}(x)=\left[s_{\mu}^{(\infty)}(x), s_{M}^{(\infty)}(x)\right]
$$

назьвается предельным интервалом Вейля. Из (26) следует включение $\{s(x)$ : $\left.s \in \mathscr{F}_{\infty}\right\} \subset \mathscr{I}^{(\infty)}(x)$. Можно доказать, что $\left\{s(x): s \in \mathscr{F}_{\infty}\right\}=\mathscr{I}^{(\infty)}(x)$.

\section{5. Критерий неопределенности проблемы моментов.}

Лемма 1. Пусть дана неопределенная усеченная проблема моментов (3) $и$ пусть $U_{1}^{(k)}$ обозначает ее резольвентную матрицу, а $s_{\mu}^{(k)} u s_{M}^{(k)}$ - соответствующие экстремальные решения. Тогда для всех $x \in \mathbb{R}_{-}$имеем

$$
\begin{aligned}
U_{1}^{(k)}(x) J_{\pi} U_{1}^{(k)^{*}}(x)-J_{\pi}=2 & {\left[\begin{array}{cc}
I & 0 \\
s_{\mu}^{(k)}(x) & I
\end{array}\right] } \\
& \times\left[\begin{array}{cc}
\left(s_{M}^{(k)}(x)-s_{\mu}^{(k)}(x)\right)^{-1} & 0 \\
0 & s_{\mu}^{(k)}(x)
\end{array}\right]\left[\begin{array}{cc}
I & s_{\mu}^{(k)}(x) \\
0 & I
\end{array}\right] .
\end{aligned}
$$


ДокАЗАТЕЛЬСтво. Для упрощения записи верхний индекс $(k)$ опускаем. Имеем

$$
\begin{aligned}
U_{1}(x) & =\left[\begin{array}{cc}
\alpha(x) & \beta(x) \\
\gamma(x) & \delta(x)
\end{array}\right]=\left[\begin{array}{cc}
I & I \\
\gamma(x) \alpha^{-1}(x) & \delta(x) \beta^{-1}(x)
\end{array}\right] \cdot\left[\begin{array}{cc}
\alpha(x) & 0 \\
0 & \beta(x)
\end{array}\right] \\
& =\left[\begin{array}{cc}
I & I \\
s_{\mu}(x) & s_{M}(x)
\end{array}\right] \cdot\left[\begin{array}{cc}
\alpha(x) \beta^{*}(x) & 0 \\
0 & I
\end{array}\right] \cdot\left[\begin{array}{cc}
\beta^{*^{-1}}(x) & 0 \\
0 & \beta(x)
\end{array}\right] \\
& =\left[\begin{array}{cc}
I & I \\
s_{\mu}(x) & s_{M}(x)
\end{array}\right] \cdot\left[\begin{array}{cc}
\left(s_{M}(x)-s_{\mu}(x)\right)^{-1} & 0 \\
0 & I
\end{array}\right] \cdot\left[\begin{array}{cc}
\beta^{*^{-1}}(x) & 0 \\
0 & \beta(x)
\end{array}\right] .
\end{aligned}
$$

В этой цепочке равенств третье следует из (14), а четвертое - из (20). Далее (для упрощения обозначений опускаем аргумент $x$ )

$$
\begin{aligned}
U_{1} J_{\pi} U_{1}^{*}-J_{\pi}= & {\left[\begin{array}{cc}
I & I \\
s_{\mu} & s_{M}
\end{array}\right]\left[\begin{array}{cc}
\left(s_{M}-s_{\mu}\right)^{-1} & 0 \\
0 & I
\end{array}\right] \cdot\left[\begin{array}{cc}
\beta^{*^{-1}} & 0 \\
0 & \beta
\end{array}\right] J_{\pi} } \\
& \times\left[\begin{array}{cc}
\beta^{-1} & 0 \\
0 & \beta^{*}
\end{array}\right]\left[\begin{array}{cc}
\left(s_{M}-s_{\mu}\right)^{-1} & 0 \\
0 & I
\end{array}\right]\left[\begin{array}{cc}
I & s_{\mu} \\
I & s_{M}
\end{array}\right]-J_{\pi} \\
= & {\left[\begin{array}{cc}
I & I \\
s_{\mu} & s_{M}
\end{array}\right]\left[\begin{array}{cc}
\left(s_{M}-s_{\mu}\right)^{-1} & 0 \\
0 & I
\end{array}\right] J_{\pi}\left[\begin{array}{cc}
\left(s_{M}-s_{\mu}\right)^{-1} & 0 \\
0 & I
\end{array}\right]\left[\begin{array}{cc}
I & s_{\mu} \\
I & s_{M}
\end{array}\right]-J_{\pi} } \\
= & {\left[\begin{array}{cc}
2\left(s_{M}-s_{\mu}\right)^{-1} & 2\left(s_{M}-s_{\mu}\right)^{-1} s_{\mu} \\
2 s_{\mu}\left(s_{M}-s_{\mu}\right)^{-1} & s_{\mu}\left(s_{M}-s_{\mu}\right)^{-1} s_{M}+s_{M}\left(s_{M}-s_{\mu}\right)^{-1} s_{\mu}
\end{array}\right] } \\
= & 2\left[\begin{array}{cc}
\left(s_{M}-s_{\mu}\right)^{-1} & \left(s_{M}-s_{\mu}\right)^{-1} s_{\mu} \\
s_{\mu}\left(s_{M}-s_{\mu}\right)^{-1} & \left(s_{\mu}^{-1}-s_{M}^{-1}\right)^{-1}
\end{array}\right] \\
= & 2\left[\begin{array}{cc}
\left(s_{M}-s_{\mu}\right)^{-1} & \left(s_{M}-s_{\mu}\right)^{-1} s_{\mu} \\
s_{\mu}\left(s_{M}-s_{\mu}\right)^{-1} & s_{\mu}\left(s_{M}-s_{\mu}\right)^{-1} s_{M}
\end{array}\right] \\
= & 2\left[\begin{array}{cc}
\left(s_{M}-s_{\mu}\right)^{-1} & \left(s_{M}-s_{\mu}\right)^{-1} s_{\mu} \\
s_{\mu}\left(s_{M}-s_{\mu}\right)^{-1} & s_{\mu}\left(s_{M}-s_{\mu}\right)^{-1} s_{\mu}+s_{\mu}
\end{array}\right] \\
= & 2\left[\begin{array}{cc}
I & 0 \\
s_{\mu} & I
\end{array}\right]\left[\begin{array}{cc}
\left(s_{M}-s_{\mu}\right)^{-1} & 0 \\
0 & s_{\mu}
\end{array}\right]\left[\begin{array}{cc}
I & s_{\mu} \\
0 & I
\end{array}\right] .
\end{aligned}
$$

Мы будем использовать хорошо известное свойство

$$
\left[\begin{array}{cc}
A & B \\
B^{*} & C
\end{array}\right]>0 \Longrightarrow\left[\begin{array}{cc}
A & B \\
B^{*} & C
\end{array}\right]^{-1} \geqslant\left[\begin{array}{cc}
A^{-1} & 0 \\
0 & 0
\end{array}\right]
$$

Лемма 2. Пусть $s_{M}^{(\infty)}, s_{\mu}^{(\infty)} \in \mathscr{F}_{\infty}$. Тогда для всех $x_{1}, x_{2} \in \mathbb{R}_{-}$имеет место равенство

$$
\operatorname{rank}\left\{s_{M}^{(\infty)}\left(x_{1}\right)-s_{\mu}^{(\infty)}\left(x_{1}\right)\right\}=\operatorname{rank}\left\{s_{M}^{(\infty)}\left(x_{2}\right)-s_{\mu}^{(\infty)}\left(x_{2}\right)\right\}
$$

ДокАЗАТЕЛЬСТво. Из (28) вытекают неравенства

$$
\begin{gathered}
{\left[\left\{s_{i+j}\right\}_{i, j=0}^{n}\right]^{-1} \geqslant\left[\begin{array}{cc}
{\left[\left\{s_{i+j}\right\}_{i, j=0}^{n-1}\right]^{-1}} & 0 \\
0 & 0
\end{array}\right],} \\
{\left[\left\{s_{i+j+1}\right\}_{i, j=0}^{n}\right]^{-1} \geqslant\left[\begin{array}{cc}
{\left[\left\{s_{i+j+1}\right\}_{i, j=0}^{n-1}\right]^{-1}} & 0 \\
0 & 0
\end{array}\right] .}
\end{gathered}
$$


Отсюда и из (11) следует, что при $k>0, \operatorname{Re} z<0$

$$
U_{1}^{(k+1)}(z) J_{\pi} U_{1}^{(k+1)^{*}}(z)-J_{\pi} \geqslant U_{1}^{(k)}(z) J_{\pi} U_{1}^{(k)^{*}}(z)-J_{\pi} \geqslant 0 .
$$

По теореме С. А. Орлова (см. [8], [9]) существуют пределы

$$
\Gamma(z):=\lim _{k \rightarrow \infty}\left[U_{1}^{(k)}(z) J_{\pi} U_{1}^{(k)^{*}}(z)-J_{\pi}\right]^{-1}, \quad \operatorname{Re} z<0,
$$

и для всех $z_{1}$ и $z_{2}$ из левой полуплоскости

$$
\operatorname{rank} \Gamma\left(z_{1}\right)=\operatorname{rank} \Gamma\left(z_{2}\right)
$$

Из формулы $(27)$ имеем $\left(\forall x \in \mathbb{R}_{-}\right)$

$$
\Gamma(x)=\frac{1}{2}\left[\begin{array}{cc}
I & -s_{\mu}^{(\infty)}(x) \\
0 & I
\end{array}\right]\left[\begin{array}{cc}
s_{M}^{(\infty)}(x)-s_{\mu}^{(\infty)}(x) & 0 \\
0 & s_{\mu}^{(\infty)^{-1}}(x)
\end{array}\right]\left[\begin{array}{cc}
I & 0 \\
-s_{\mu}^{(\infty)}(x) & I
\end{array}\right] .
$$

Следовательно, $\operatorname{rank} \Gamma(x)=\operatorname{rank}\left[s_{M}^{(\infty)}(x)-s_{\mu}^{(\infty)}(x)\right]+m$. Отсюда и из $(32)$ следует $(29)$.

ОПРЕДЕЛЕНИЕ 5. Проблема моментов Стилтьеса (1) назьвается неопределенной, если все предельные интервалы Вейля

$$
\mathscr{I}^{(\infty)}(x)=\left[s_{\mu}^{(\infty)}(x), s_{M}^{(\infty)}(x)\right] \quad \forall x \in \mathbb{R}_{-}
$$

являются невырожденными матричными интервалами.

Из этого определения и леммы 2 непосредственно вытекает

ЛЕмма 3. Для того чтобы проблема моментов Стилтьеса (1) была неопределенной, необходимо, чтобы при всех $x \in \mathbb{R}_{-}$выполнялось неравенство

$$
s_{\mu}^{(\infty)}(x)<s_{M}^{(\infty)}(x)
$$

и достаточно, чтобы неравенство (33) выполнялось хотя бы для одного $x \in \mathbb{R}_{-}$.

Другими словами, для неопределенности проблемы (1) необходимо, чтобы при всех $x \in \mathbb{R}_{-}$предельные интервалы Вейля были невырожддены, и достаточно, чтобы хотя бы для одного $x \in \mathbb{R}_{-}$был невырохсденым соответствующий предельный интервал Вейля.

ТЕорема 6. Для того, чтобы проблема моментов Стилтьеса (1) была неопределенной, необходимо и достаточно, чтобы в любой фиксированной точке $x_{0} \in \mathbb{R}_{-}$существовали предель

$$
\begin{aligned}
& \lim _{k \rightarrow \infty}\left[-x_{0} v_{1}^{(k)^{*}} R_{T_{1}^{*}}^{(k)}\left(x_{0}\right) K_{1}^{(k)^{-1}} R_{T_{1}}^{(k)}\left(x_{0}\right) v_{1}^{(k)}\right]>0, \\
& \lim _{k \rightarrow \infty}\left[x_{0}^{2} v_{2}^{(k)^{*}} R_{T_{2}^{*}}^{(k)}\left(x_{0}\right) K_{2}^{(k)^{-1}} R_{T_{2}}^{(k)}\left(x_{0}\right) v_{2}^{(k)}\right]>0 .
\end{aligned}
$$


ДокАЗАТЕЛЬСТво. Из (30) следует, что положительно определенные матрицы

$$
-x_{0} v_{1}^{(k)^{*}} R_{T_{1}^{*}}^{(k)}\left(x_{0}\right) K_{1}^{(k)^{-1}} R_{T_{1}}^{(k)}\left(x_{0}\right) v_{1}^{(k)}, \quad x_{0}^{2} v_{2}^{(k)^{*}} R_{T_{2}^{*}}^{(k)}\left(x_{0}\right) K_{2}^{(k)^{-1}} R_{T_{2}}^{(k)}\left(x_{0}\right) v_{2}^{(k)}
$$

монотонно возрастают с ростом $k$.

Пусть теперь проблема моментов (1) является неопределенной. Для $k$-й усеченной проблемы моментов (3) запишем (16) при $z=x_{0} \in \mathbb{R}_{-}$. Получим

$$
\begin{aligned}
\left\{s_{M}^{(k)}\left(x_{0}\right)-s_{\mu}^{(k)}\left(x_{0}\right)\right\}^{-1}= & -x_{0} v_{1}^{(k)^{*}} R_{T_{1}^{*}}^{(k)}\left(x_{0}\right) K_{1}^{(k)^{-1}} R_{T_{1}}^{(k)}\left(x_{0}\right) v_{1}^{(k)} \\
& +x_{0}^{2} v_{2}^{(k)^{*}} R_{T_{2}^{*}}^{(k)}\left(x_{0}\right) K_{2}^{(k)^{-1}} R_{T_{2}}^{(k)}\left(x_{0}\right) v_{2}^{(k)} .
\end{aligned}
$$

Оба слагаемых в правой части последнего равенства являются положительно определенными матрицами и монотонно возрастают с ростом $k$. Левая часть в силу неопределенности проблемы моментов при $k \rightarrow+\infty$ стремится к положительно определенной матрице $\left\{s_{M}^{(\infty)}\left(x_{0}\right)-s_{\mu}^{(\infty)}\left(x_{0}\right)\right\}^{-1}$. Отсюда следует существование и строгая положительность пределов в (34).

Наоборот, пусть существуют и строго положительны оба предела в (34). Совершим теперь предельный переход $k \rightarrow+\infty$ в обеих частях равенства (35). Получим, что $\left\{s_{M}^{(\infty)}\left(x_{0}\right)-s_{\mu}^{(\infty)}\left(x_{0}\right)\right\}^{-1}>0$. Таким образом, предельньй интервал Вейля $\mathscr{I}^{(\infty)}\left(x_{0}\right)$ является невырожденным и, следовательно, проблема моментов (1) является неопределенной.

6. Критерий Стилтьеса. Имеет место теорема (см. [10], [11]).

Теорема 7. Резольвентные матрииы усеченных проблем моментов (3) разбиваются в произведение Бляике-Потапова

$$
\begin{gathered}
U_{1}^{(2 n)}(z)=\left[\begin{array}{cc}
I & -z M_{0} \\
0 & I
\end{array}\right] \cdot\left[\begin{array}{cc}
I & 0 \\
L_{0} & I
\end{array}\right] \cdots\left[\begin{array}{cc}
I & 0 \\
L_{n-1} & I
\end{array}\right] \cdot\left[\begin{array}{cc}
I & -z M_{n} \\
0 & I
\end{array}\right], \\
U_{1}^{(2 n+1)}(z)=\left[\begin{array}{cc}
I & -z M_{0} \\
0 & I
\end{array}\right] \cdot\left[\begin{array}{cc}
I & 0 \\
L_{0} & I
\end{array}\right] \cdots\left[\begin{array}{cc}
I & -z M_{n} \\
0 & I
\end{array}\right] \cdot\left[\begin{array}{cc}
I & 0 \\
L_{n} & I
\end{array}\right] .
\end{gathered}
$$

Здеcb

$$
\begin{aligned}
& M_{0}=s_{0}^{-1}>0, \quad L_{0}=s_{0} s_{1}^{-1} s_{0}>0, \\
& M_{j}=v_{1}^{(2 j)^{*}} K_{1}^{(2 j)^{-1}} v_{1}^{(2 j)}-v_{1}^{(2 j-2)^{*}} K_{1}^{(2 j-2)^{-1}} v_{1}^{(2 j-2)}>0, \\
& L_{j}=u_{2}^{(2 j+1)^{*}} K_{2}^{(2 j+1)^{-1}} u_{2}^{(2 j+1)}-u_{2}^{(2 j-1)^{*}} K_{2}^{(2 j-1)^{-1}} u_{2}^{(2 j-1)}>0
\end{aligned}
$$

для $в$ сех $j \geqslant 1$.

Параметры $M_{j}$ и $L_{j}$ назьваются параметрами Стилтьеса. Множители Бляшке-Потапова допускают экспоненциальные представления

$$
\left[\begin{array}{cc}
I & -z M_{j} \\
0 & I
\end{array}\right]=\exp \left[\begin{array}{cc}
0 & -z M_{j} \\
0 & 0
\end{array}\right], \quad\left[\begin{array}{cc}
I & 0 \\
L_{j} & I
\end{array}\right]=\exp \left[\begin{array}{cc}
0 & 0 \\
L_{j} & 0
\end{array}\right], \quad j \geqslant 0 .
$$

Тогда резольвентную матрицу можно записать в виде $(j \geqslant 0)$

$$
U_{1}^{(k)}(z)=\prod_{j=0}^{k} e^{H_{j}(z)}, \quad H_{2 j}(z)=\left[\begin{array}{cc}
0 & -z M_{j} \\
0 & 0
\end{array}\right], \quad H_{2 j+1}(z) \equiv\left[\begin{array}{cc}
0 & 0 \\
L_{j} & 0
\end{array}\right] .
$$

В матричных произведениях считаем, что индексы сомножителей возрастают в порядке слева на право. 
Теорема 8. Для того, чтобы проблема моментов Стилтьеса (1) была неопределенной, необходимо и достаточно, чтобь сходились ряды

$$
\sum_{j=0}^{\infty} M_{j} \quad u \quad \sum_{j=0}^{\infty} L_{j} .
$$

ДокАЗАТЕЛЬСтво. Пусть проблема (1) является неопределенной. Покажем, что для всех $k \geqslant 1$ вьполнены следующие три соотношения:

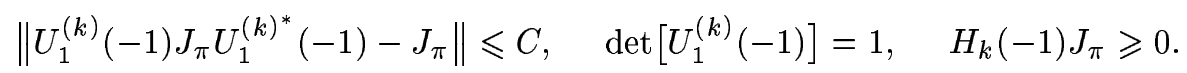

Здесь $C \in \mathbb{R}_{+}-$некоторая константа. Действительно, из (27), (33) и (22) следует сходимость $U_{1}^{(k)}(-1) J_{\pi} U_{1}^{(k)^{*}}(-1)-J_{\pi}$ при $k \rightarrow \infty$. Отсюда следует первое из соотношений (40). Равенство в (40) следует из (36). С учетом (38) имеем

$$
H_{2 j}(-1) J_{\pi}=\left[\begin{array}{cc}
M_{j} & 0 \\
0 & 0
\end{array}\right] \geqslant 0, \quad H_{2 j+1}(-1) J_{\pi}=\left[\begin{array}{cc}
0 & 0 \\
0 & L_{j}
\end{array}\right] \geqslant 0 .
$$

Доказаны все соотношения (40). По теореме Потапова (см. [12]) сходится матричное произведение $\prod_{j=0}^{\infty} e^{H_{j}(-1)}$ и ряд $\sum_{j=0}^{\infty} H_{j}(-1)$. Из сходимости последнего ряда и из вида $H_{j}(-1)$ следует сходимость рядов (39).

Наоборот, пусть сходятся ряды (39). Отсюда и из (38) следует сходимость ряда $\sum_{j=0}^{\infty} H_{j}(-1)$. Поэтому (см. [12]) сушествует предел

$$
U_{1}^{(\infty)}(-1)=\lim _{k \rightarrow \infty} e^{H_{0}(-1)} e^{H_{1}(-1)} \ldots e^{H_{k}(-1)} .
$$

Из (27) следует

$$
\begin{aligned}
& {\left[\begin{array}{cc}
s_{M}^{(k)}(-1)-s_{\mu}^{(k)}(-1) & 0 \\
0 & s_{\mu}^{(k)^{-1}}(-1)
\end{array}\right]\left[\begin{array}{cc}
I & 0 \\
-s_{\mu}^{(k)}(-1) & I
\end{array}\right]} \\
& \quad \times\left[U_{1}^{(k)}(-1) J_{\pi} U_{1}^{(k)^{*}}(-1)-J_{\pi}\right]=2\left[\begin{array}{cc}
I & s_{\mu}^{(k)}(-1) \\
0 & I
\end{array}\right] .
\end{aligned}
$$

В этом равенстве все матрицы имеют пределы при $k \rightarrow \infty$, а предел правой части является невырожденной матрицей. Поэтому $s_{M}^{(\infty)}(-1)-s_{\mu}^{(\infty)}(-1)>0$, т.е. проблема моментов (1) является неопределенной.

7. Аналитические свойства резольвентной матрицы. Нетрудно показать, что $\forall A_{0}, A_{1}, \ldots, A_{k} \in \mathbb{C}^{m \times m}$ имеют место неравенства

$$
\begin{aligned}
\left\|e^{A_{0}} e^{A_{1}} \ldots e^{A_{k}}\right\| & \leqslant \exp \left(\sum_{j=0}^{k}\left\|A_{j}\right\|\right) \\
\left\|e^{A_{0}} e^{A_{1}} \ldots e^{A_{k}}-I\right\| & \leqslant\left(\sum_{j=0}^{k}\left\|A_{j}\right\|\right) \cdot \exp \left(\sum_{j=0}^{k}\left\|A_{j}\right\|\right) .
\end{aligned}
$$


ТЕоремА 9. Пусть дана неопределенная проблема моментов (1) и пусть последовательности $\mathrm{M} \Phi\left\{U_{1}^{(k)}\right\}_{k=0}^{+\infty} u\left\{U_{2}^{(k)}\right\}_{k=0}^{+\infty}$ определены формулами (6) $u$ (7). Тогда существуют иелые $\mathrm{M} \Phi U_{1}^{(\infty)}, U_{2}^{(\infty)}: \mathbb{C} \rightarrow \mathbb{C}^{2 m \times 2 m}$ такие, ито существуют равномерные на компактах $K \subset \mathbb{C}$ предель

$$
\lim _{k \rightarrow+\infty} U_{1}^{(k)}(z)=U_{1}^{(\infty)}(z), \quad \lim _{k \rightarrow+\infty} U_{2}^{(k)}(z)=U_{2}^{(\infty)}(z) \quad \forall z \in \mathbb{C},
$$

Более того, $\operatorname{det} U_{1}^{(\infty)}(z) \equiv \operatorname{det} U_{2}^{(\infty)}(z) \equiv 1 \forall z \in \mathbb{C}$.

ДокАЗАТЕльство. Пусть резольвентные матрицы усеченных задач (3) записаны в виде (38) и пусть, для определенности, $l \geqslant k$. Тогда, воспользовавшись неравенствами (41) и сходимостью рядов (39), получим

$$
\begin{aligned}
& \left\|U_{1}^{(l)}(z)-U_{1}^{(k)}(z)\right\| \leqslant\left\|e^{H_{0}} e^{H_{1}} \ldots e^{H_{k}}\right\| \cdot\left\|e^{H_{k+1}} e^{H_{k+2}} \ldots e^{H_{l}}-I\right\| \\
& \leqslant \exp \left(\sum_{j=0}^{k}\left\|H_{j}\right\|\right) \cdot \exp \left(\sum_{j=k+1}^{l}\left\|H_{j}\right\|\right) \cdot\left(\sum_{j=k+1}^{l}\left\|H_{j}\right\|\right) \\
& \leqslant \underbrace{\exp \left(|z| \sum_{j=0}^{\infty}\left\|\left[\begin{array}{cc}
0 & M_{j} \\
0 & 0
\end{array}\right]\right\|\right) \cdot \exp \left(\sum_{j=0}^{\infty}\left\|\left[\begin{array}{cc}
0 & 0 \\
L_{j} & 0
\end{array}\right]\right\|\right)}_{\Phi(z)} \cdot\left(\sum_{j=k+1}^{l}\left\|H_{j}\right\|\right) \\
& =\Phi(z) \cdot\left(|z| \sum_{j \in[k+1, l]}^{+}\left\|\left[\begin{array}{cc}
0 & M_{\frac{j}{2}} \\
0 & 0
\end{array}\right]\right\|+\sum_{j \in[k+1, l]}^{-}\left\|\left[\begin{array}{cc}
0 & 0 \\
L_{\frac{j-1}{2}} & 0
\end{array}\right]\right\|\right) .
\end{aligned}
$$

Здесь символы $\sum^{+}$и $\sum^{-}$обозначают соответственно суммирование по всем четным или нечетным значениям индекса $j$. Определенная в последних равенствах функция $\Phi$ : $\mathbb{C} \rightarrow \mathbb{R}_{+}$непрерывна. Пусть теперь задан произвольньй компакт $K \subset \mathbb{C}$ и пусть $C=$ $\max _{z \in K}|\Phi(z)|$ и $c=\max _{z \in K}|z|$. Тогда

$$
\left\|U_{1}^{(l)}(z)-U_{1}^{(k)}(z)\right\| \leqslant C\left(c \sum_{j \in[k+1, l]}^{+}\left\|\left[\begin{array}{cc}
0 & M_{\frac{j}{2}} \\
0 & 0
\end{array}\right]\right\|+\sum_{j \in[k+1, l]}^{-}\left\|\left[\begin{array}{cc}
0 & 0 \\
L_{\frac{j-1}{2}} & 0
\end{array}\right]\right\|\right) .
$$

Учитьвая сходимость рядов (39), получаем, что последовательность резольвентных матриц $\left\{U_{1}^{(k)}(z)\right\}_{k=0}^{\infty}$ равномерно фундаментальна на компактах из $\mathbb{C}$. Осталось заметить, что элементы резольвентных матриц $\left\{U_{1}^{(k)}(z)\right\}_{k=0}^{\infty}$ являются полиномами по $z$. Существование второго предела в (42) доказьвается аналогичньп образом.

Из (36) следует, что $\operatorname{det} U_{1}^{(k)}(z) \equiv 1$. Совершая предельный переход $k \rightarrow \infty$, получим $\operatorname{det} U_{1}^{(\infty)}(z) \equiv 1$. Утверждение относительно детерминанта $U_{2}^{(\infty)}$ вытекает из очевидного тождества

$$
U_{2}^{(k)}(z)=\left[\begin{array}{cc}
z^{-1} I & 0 \\
0 & I
\end{array}\right] U_{1}^{(k)}(z)\left[\begin{array}{cc}
z I & 0 \\
0 & I
\end{array}\right]
$$

ОПрЕДЕЛЕниЕ $6 . \mathrm{M} \Phi U_{1}^{(\infty)}: \mathbb{C} \rightarrow \mathbb{C}^{2 m \times 2 m}$, определенная в $(42)$, назьвается резольвентной матрицей неопределенной проблемы моментов (1). 
ТЕОРемА 10. Пусть проблема моментов (1) является неопределенной и пусть, далее, $U_{1}^{(\infty)}: \mathbb{C} \rightarrow \mathbb{C}^{2 m \times 2 m}$ - ее резольвентная матрича. Тогда для любого $\varepsilon>0$ существует зависящая только от в константа $C_{\varepsilon}$ такая, что

$$
\left\|U_{1}^{(\infty)}(z)\right\| \leqslant C_{\varepsilon} \exp (\varepsilon|z|) \quad \forall z \in \mathbb{C} .
$$

Другими словами, әлементы резольвентной матрицы $U_{1}$ являются иелыми функииями не выше первого порядка минимального әкспоненциального типа.

ДокАЗАтЕЛЬСтво. Из неопределенности проблемы моментов (1) вытекает сходимость рядов (39). Но тогда сходятся ряды $\sum_{j=0}^{\infty}\left\|M_{j}\right\|$ и $\sum_{j=0}^{\infty}\left\|L_{j}\right\|$. Пусть дано некоторое $\varepsilon>0$. Тогда существует $N_{\varepsilon}$ такое, что для всех $k>N_{\varepsilon}$ вьполняется неравенство

$$
\sum_{j \in\left[N_{\varepsilon}+1, k\right]}^{+}\left\|\left[\begin{array}{cc}
0 & M_{\frac{j}{2}} \\
0 & 0
\end{array}\right]\right\|<\frac{\varepsilon}{2} .
$$

Оценим норму резольвентной матрицы $U_{1}^{(k)}$ :

$$
\begin{aligned}
\left\|U_{1}^{(k)}(z)\right\| & =\left\|e^{H_{0}(z)} e^{H_{1}(z)} \ldots e^{H_{k}(z)}\right\| \\
& \leqslant\left\|e^{H_{0}(z)} e^{H_{1}(z)} \ldots e^{H_{N_{\varepsilon}}(z)}\right\| \cdot\left\|e^{H_{N_{\varepsilon}+1}(z)} \ldots e^{H_{k}(z)}\right\| \\
& =\left\|\left(I+H_{0}(z)\right)\left(I+H_{1}(z)\right) \ldots\left(I+H_{N_{\varepsilon}}(z)\right)\right\| \cdot\left\|e^{H_{N_{\varepsilon}+1}(z)} \ldots e^{H_{k}(z)}\right\| \\
& \leqslant\left(1+\left\|H_{0}(z)\right\|\right)\left(1+\left\|H_{1}(z)\right\|\right) \ldots\left(1+\left\|H_{N_{\varepsilon}}(z)\right\|\right) \cdot\left\|e^{H_{N_{\varepsilon}+1}(z)} \ldots e^{H_{k}(z)}\right\| \\
& =P(|z|) \cdot\left\|e^{H_{N_{\varepsilon}+1}(z)} \ldots e^{H_{k}(z)}\right\| .
\end{aligned}
$$

Здесь $P$ - многочлен с положительными коэффициентами (см. вид матриц $H_{j}$ в $(38)$ ). Сушествует зависящая только от $\varepsilon$ константа $A_{\varepsilon}$ такая, что

$$
P(|z|) \leqslant A_{\varepsilon} \exp \left(\frac{\varepsilon|z|}{2}\right) \quad \forall z \in \mathbb{C} .
$$

Таким образом,

$$
\begin{aligned}
& \left\|U_{1}^{(k)}(z)\right\| \leqslant P(|z|) \cdot\left\|e^{H_{N_{\varepsilon}+1}(z)} \ldots e^{H_{k}(z)}\right\| \\
& \leqslant A_{\varepsilon} \cdot \exp \left(\frac{\varepsilon|z|}{2}\right) \cdot\left\|e^{H_{N_{\varepsilon}+1}(z)} \ldots e^{H_{k}(z)}\right\| \\
& \leqslant A_{\varepsilon} \cdot \exp \left(\frac{\varepsilon|z|}{2}\right) \cdot \exp \left(\sum_{j \in\left[N_{\varepsilon}+1, k\right]}\left\|H_{j}(z)\right\|\right) \\
& =A_{\varepsilon} \exp \left(\frac{\varepsilon|z|}{2}\right) \cdot \exp \left(\sum_{j \in\left[N_{\varepsilon}+1, k\right]}^{+}\left\|H_{j}(z)\right\|\right) \cdot \exp \left(\sum_{j \in\left[N_{\varepsilon}+1, k\right]}^{-}\left\|H_{j}(z)\right\|\right) \\
& =A_{\varepsilon} \cdot \exp \left(\frac{\varepsilon|z|}{2}\right) \cdot \exp \left(\underset{j \in\left[\sum_{\varepsilon}+1, k\right]}{+}\left\|\left[\begin{array}{cc}
0 & M_{\frac{j}{2}} \\
0 & 0
\end{array}\right]\right\|\right) \\
& \times \exp \left(\sum_{j \in\left[N_{\varepsilon}+1, k\right]}^{-}\left\|\left[\begin{array}{cc}
0 & 0 \\
L_{\frac{j-1}{2}} & 0
\end{array}\right]\right\|\right) \leqslant \\
& \leqslant A_{\varepsilon} \cdot \exp \left(\sum_{j=0}^{\infty}\left\|\left[\begin{array}{cc}
0 & 0 \\
L_{j} & 0
\end{array}\right]\right\|\right) \cdot \exp \left(\frac{\varepsilon|z|}{2}\right) \cdot \exp \left(\frac{\varepsilon|z|}{2}\right) \\
& =C_{\varepsilon} \cdot \exp (\varepsilon|z|), \quad C_{\varepsilon}=A_{\varepsilon} \cdot \exp \left(\sum_{j=0}^{\infty}\left\|\left[\begin{array}{cc}
0 & 0 \\
L_{j} & 0
\end{array}\right]\right\|\right) \text {. }
\end{aligned}
$$


В этой цепочке равенств и неравенств третье вытекает из (41), пятое - из (38), шестое из сходимости ряда $\sum_{j=0}^{\infty}\left\|L_{j}\right\|$ и неравенства $(44)$.

Итак, мы доказали неравенство $\left\|U_{1}^{(k)}(z)\right\| \leqslant C_{\varepsilon} \cdot \exp (\varepsilon|z|)$. Переходя в этом неравенстве к пределу при $k \rightarrow \infty$, получим (43).

\section{8. Описание множества решений проблемы моментов.}

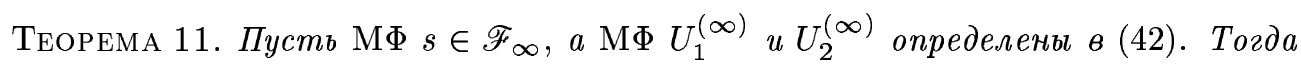
$\forall z \in \mathbb{C}_{ \pm}$выполнены основные матричные неравенства

$$
\left[I \bar{z}^{r-1} s^{*}(z)\right] \frac{U_{r}^{(\infty)^{-1^{*}}}(z) J U_{r}^{(\infty)^{-1}}(z)}{i(\bar{z}-z)}\left[\begin{array}{c}
I \\
z^{r-1} s(z)
\end{array}\right] \geqslant 0, \quad z \in \mathbb{C}_{ \pm}, \quad r=1,2 .
$$

Наоборот, пусть для некоторой голоморфной в $\mathbb{C}_{+} \mathrm{M} \Phi$ s неравенства (45) выполняются хотя бъ $\forall z \in \mathbb{C}_{+}$. Тогда $s$ допускает представление (2) и участвующая в этом представлении $\mathrm{M} \Phi \sigma \in \mathscr{M}_{\infty}$, m.e. $s \in \mathscr{F}_{\infty}$.

ДокАЗАтЕльство. Пусть $s \in \mathscr{F}_{\infty}$. Тогда для всех $k>0 \mathrm{M} \Phi s \in \mathscr{F}_{k}$. По теореме 1 рассматриваемая М $\Phi$ удовлетворяет системе ОМН

$$
\left[I \bar{z}^{r-1} s^{*}(z)\right] \frac{U_{r}^{(k)^{-1}}(z) J U_{r}^{(k)^{-1}}(z)}{i(\bar{z}-z)}\left[\begin{array}{c}
I \\
z^{r-1} s(z)
\end{array}\right] \geqslant 0, \quad z \in \mathbb{C}_{ \pm}, \quad r=1,2 .
$$

Переходя к пределу $k \rightarrow \infty$, получим неравенство (45).

Наоборот, пусть М $\Phi$ голоморфна в $\mathbb{C}_{+}$и удовлетворяет там системе ОМН (45). Из (10) и (28) вытекает неравенство

$$
\frac{U_{r}^{(k)^{-1^{*}}}(z) J U_{r}^{(k)^{-1}}(z)}{i(\bar{z}-z)} \geqslant \frac{U_{r}^{(l)^{-1^{*}}}(z) J U_{r}^{(l)^{-1}}(z)}{i(\bar{z}-z)}, \quad z \in \mathbb{C}_{+}, \quad r=1,2, \quad l>k .
$$

Переходя в этом неравенстве к пределу при $l \rightarrow \infty$, получим

$$
\frac{U_{r}^{(k)^{-1^{*}}}(z) J U_{r}^{(k)^{-1}}(z)}{i(\bar{z}-z)} \geqslant \frac{U_{r}^{(\infty)^{-1^{*}}}(z) J U_{r}^{(\infty)^{-1}}(z)}{i(\bar{z}-z)}, \quad z \in \mathbb{C}_{+}, \quad r=1,2 .
$$

Умножим обе части неравенства слева на матрищу $\left[I \bar{z}^{r-1} s^{*}(z)\right]$, а справа на сопряженную матрицу. С учетом (45) получим

$$
\left[I \bar{z}^{r-1} s^{*}(z)\right] \frac{U_{r}^{(k)^{-1^{*}}}(z) J U_{r}^{(k)^{-1}}(z)}{i(\bar{z}-z)}\left[\begin{array}{c}
I \\
z^{r-1} s(z)
\end{array}\right] \geqslant 0, \quad z \in \mathbb{C}_{+}, \quad r=1,2 .
$$

По теореме $1 s \in \mathscr{F}_{k}$. В силу произвольности $k$ имеем $s \in \mathscr{F}_{\infty}$.

Доказательство следующей теоремы опирается на систему ОМН (45) и проводится по аналогии с доказательством аналогичных утверждений в [5], [13] и [14].

ТЕОРемА 12. Пусть проблема моментов (1) является неопределенной и резольвентная матрица $U_{1}^{(\infty)}$ разбита на четыре $m \times m$ блока $\alpha^{(\infty)}, \beta^{(\infty)}, \gamma^{(\infty)}, \delta^{(\infty)}$. Тогда формула

$$
s(z)=\frac{\gamma^{(\infty)}(z) p(z)+\delta^{(\infty)}(z) q(z)}{\alpha^{(\infty)}(z) p(z)+\beta^{(\infty)}(z) q(z)}
$$

устанавливает биективное соответствие меэду $\mathscr{F}_{\infty}$ и $\mathscr{S}_{\infty}$. 


\section{СПИСОК ЦИТИРОВАННОЙ ЛИТЕРАТУРЫ}

[1] Stieltjes T. Recherches sur les fractions continues // Anns. Fac. Sci. Univ. Toulouse. 1894. № 8. P. 1-122; 1895. №9. P. 1-47.

[2] Simon B. The classical moment problem as a self-adjoint finite difference operator // Adv. in Math. 1998. V. 137. P. 82-203.

[3] Крейн М. Г., Нудельман А. А. Проблема моментов Маркова и экстремальные задачи. М.: Наука, 1973.

[4] Дюкарев Ю. М. Общая схема решения интерполяционных задач в классе Стилтьеса, основанная на согласованных интегральных представлениях пар неотрицательных операторов. 1 // Матем. физика, анализ, геометрия. 1999. Т. 6. №1/2. С. 30-54.

[5] Дюкарев Ю. М., Чоке Риверо А. Е. Степенная проблема моментов на компактном интервале // Матем. заметки. 2001. Т. 69. № 2. С. 200-213.

[6] Потапов В. П. Дробно-линейные преобразования матриц // Исследования по теории операторов и их приложениям. Киев: Наукова думка, 1979. С. 75-97.

[7] Donoghue W. F. Monotone Matrix Functions and Analytic Continuation. Springer-Verlag, 1974.

[8] Орлов С. А. Гнездящиеся матричные круги, аналитически зависящие от параметра и теоремы об инвариантности рангов радиусов предельных матричных кругов // Изв. АН СССР. Сер. матем. 1976. Т. 40. №3. С. 593-644.

[9] Потапов В. П. К теории матричных кругов Вейля // Функциональный анализ и прикладная математика. Киев: Наукова думка, 1982. С. 113-121.

[10] Дюкарев Ю. М. Факторизация оператор-функций мультипликативного класса Стилтьеса // Докл. НАН Украины. 2000. № 9. С. 23-26.

[11] Дюкарев Ю. М. Мультипликативная структура резольентных матриц интерполяионных задач в классе Стилтьеса // Вестн. Харьковского ун-та. Сер. Матем., прикладная матем. и мех. 1999. № 458. С. 143-153.

[12] Потапов В. П. Теорема о модуле // Теория функций, функцион. анализ и их прилож. 1983. № 39. С. $95-106$.

[13] Дюкарев Ю. М., Кацнельсон В. Э. Мультипликативные и аддитивные классы Стилтьеса аналитических матриц-функций и связанные с ними интерполяционные задачи // Теория функций, функцион. анализ и их прилож. 1981. №36. С. 13-27.

[14] Bolotnikov V., Sakhnovich L. On an operator approach to interpolation problems for Stieltjes functions // Integral Equations Operator Theory. 1999. № 35. P. 423-470.

Харьковский национальный университет

Поступило

E-mail: Yury.M.Dyukarev@univer.kharkov.ua

08.10 .2002

Исправленный вариант

13.03.2003 